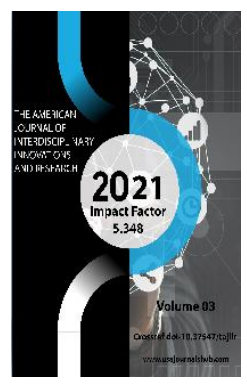

Copyright: Original content from this work may be used under the terms of the creative commons attributes 4.0 licence.

\section{Boundary Action On Simple Reduced Group C*-Algebras}

\author{
V.A. Onuche \\ Department Of Mathematics, University Of Uyo, Akwa Ibom State, Nigeria \\ T.J Alabiand \\ Department Of Mathematics, Kogi State Polytechnic Lokoja, Lokoja Nigeria \\ O.F. Ajayi \\ Department Of Computer Science, Kogi State Polytechnic Lokoja, Lokoja Nigeria
}

\title{
ABSTRACT
}

A connection between boundary actions, ideal structure of reduced crossed products and $\mathrm{C}^{*}$-simple group is imminent.We investigate the stability properties for discrete group pioneered by powers and show that the non-abelian free group on two generators is $C^{*}$-simple.Kalantar and Kennedy [32, Theorem 6.2] is now extended. Some examples are given using characterization of $C^{*}$-simplicity obtained by Kalantar, Kennedy, Breuillard, and Ozawa [10, Theorem 3.1]

\section{KEYWORDS}

Boundaries, $c^{*}$-algebra of discrete group, simple group

\section{INTRODUCTION}

Let $G$ be a discrete group. Let the group algebra $I_{1}(G)$ equipped with the following product and involution 


$$
(x y)(s)=\sum x(g) y(g-1 s), x_{i}(s)={ }^{\prime} x(s-1), x, y \in l 1(G), s \in G
$$

This product is known as the convolution of two functions $x, y: G \rightarrow C$ with respect to these operations and the usual 1 - norm, $l(G)$ is a Banach *algebra with identity $\delta 1$. The characteristic function $\delta g \in l_{1}(G)$ satisfy $\delta g \delta g i=\delta g i \delta g=\delta 1$, the self adjoint subalgebra $c c(G)$ of finitely supported function on $(G)$ constitute a dense subsect of $\left.\mathrm{I}_{(\mathrm{G}} \mathrm{G}\right)$. It is clear that any *-homomorphism from a Banach *-algebra

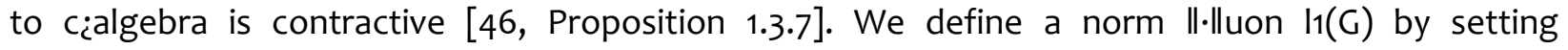
$\|x\|_{u}=\|\pi(x)\|_{\dot{c}}$ for $x \in l_{1}(G)$ where $\pi$ runs through all non-degenerate representations of $I_{1}(G)$ on a Hilbert space. Completing $I_{1}(G)$ with respect to $\|\cdot\| u$, we obtain the unital $c_{i}$-algebra, as the full group $c_{i^{-}}$ algebra denoted by $c_{i}(G)$. It is well known that any non-degenerate representation of $\mathrm{I}_{1}(\mathrm{G})$ on a Hilbert space $\mathrm{H}$ extends to a non-degenerate representation of $\mathrm{c}_{\dot{c}}(\mathrm{G})$ on $\mathrm{H}$. Thus, this correspondence of representation is one-to-one.A unitary representation of $\mathrm{G}$ is a group homomorphism of $\mathrm{G}$ into the group $\mathrm{U}(\mathrm{H})$ of unitary operators on some on some Hilbert space $\mathrm{H}$. There is one-to-one correspondence between unitary representations of $G$ and non-degenerate representations ofl1(G), given by mapping nonlinear function to the operator respectively

$$
\begin{gathered}
f=\sum_{g \in G} f|g| \delta_{g} \in l^{1}(G) \\
\sum_{g \in G} f(g) \pi_{g} \in B(H)
\end{gathered}
$$

Where $\pi: g \rightarrow \pi g$ is a unitary representation of $G$ on the Hilbert space $\mathrm{H}$. Precisely, any unitary representation of $G$ can be used to construct a $c_{i}$-algebra. Indeed, if $\pi: c_{i}(G) \rightarrow B(H)$ is the nondegenerate representation induced by a unitary representation $\pi: G \rightarrow U(H)$, then thec $c^{-}$-algebra associated to $\pi$ is given by $c_{i}(G)=\pi\left(c_{i}(G)\right)$. Next, we considerregular (left) regular representation $\lambda$ in the unitary group of $\mathrm{I}_{2}(\mathrm{G})$ given by left translation:

$$
[\lambda g \xi](s)=\xi(g-1 s), g, s \in G, \xi \in l 2(G)
$$

With respect to the canonical orthogonal basis $\{\delta 1 \mid s \in G\}$ ofl2(G), where $\lambda$ satisfies $\lambda g \delta s=\delta g s, g, s \in G$. The reduced group $c_{i}$-algebracr $i(G)$ is the $c_{i}$-algebra $c \lambda_{i}(G)$ associated to $\lambda$, and $\mathrm{cr} i(G)$ is therefore the norm-closure inB $(\mathrm{I} 2(\mathrm{G}))$ of the set of operator of the form $\Sigma \mathrm{g} \in \mathrm{G} \eta \mathrm{g} \lambda \mathrm{g}, \eta \mathrm{ng} \in \mathrm{Ca}$ non-zero for finitely manyg $\in G$. Moreover, $\mathrm{cr}_{c}(G)$ is equipped with a faithful tracial state $\tau$, given by $\tau(x)=\langle x \delta 1, \delta 1\rangle$. We refer to $\tau$ as the canonical tracial state on $\mathrm{Cr}_{i}(G)$.

Definition 1.1 A discrete group $G$ is said to bec $c_{i}$-simple if $\mathrm{cr}_{i}(G)$ is simple $c_{i}$-algebra with unique trace property andcr $i(G)$ admits tracial state.

The theory of simple $c_{i}$-algebra first introduced by Bédos[3] was later reconstructed by De La Harp [18, pp. 13]. Since then, many mathematical philosophers had made huge progress (see. [13, 16, 21, 33] etc.) 
C*-Simplicity and Boundary Actions ([32]): Letl1 $(G, A)$ denote the space of functions $x: G \rightarrow A$ satisfying $\sum g \in G\|x(g)\|<\infty$. From now on, we write the notation $x=\sum g \in G \times g \delta g$ for a function $x \in l_{1}(G, A)$, where $x g=x(g)$ for $g \in G$. We next equip $\mathrm{I}(\mathrm{G}, \mathrm{A})$ with a product and involution by defining

$$
(x y)(s)=\sum g \in G x(g)(g y(g-1 s)), x_{i}(s)=s x(s-1)
$$

So that $11(G, A)$ becomes a Banach *-algebra in the 1-norm. We identify $A$ with the image of $A$ under the *-homomorphism $a \mapsto a \delta 1$. Obviously, the subset $c c(G, A)$ of finitely supported functors $G \rightarrow A$ is a dense *-subalgebra of $\mathrm{I}(\mathrm{G}, \mathrm{A})$ and that an approximate identity (ei) in $\mathrm{A}$ yields an approximate identity $($ ei $\delta 1) \in l_{1}(G, A)$. It is well known that a covariant representation of the $c^{*}$-dynamical system $(A, G, a)$ is a triple $(\pi, u, H)$, where $H$ is a Hilbert space, $\pi: A \rightarrow B(H)$ is a non-degenerate representation and $\mathrm{u}: \mathrm{G} \rightarrow \mathrm{U}(\mathrm{H})$ is a unitary representation such that $\pi(\mathrm{ga})=$ ugaug $i$ for $\mathrm{g} \in \mathrm{G}$ and $\mathrm{a} \in \mathrm{A}$. We often suppress the Hilbert space $\mathrm{H}$ from the notationif is clear from the context. The associated integral form of covariant representation $(\pi, u)$ is the map $\pi \times \mathrm{u}: \mathrm{l}(\mathrm{G}, \mathrm{A}) \rightarrow \mathrm{B}(\mathrm{H})$ define by

$$
(\pi \times \mathrm{u})(\mathrm{x})=\sum \mathrm{g} \in \mathrm{G} \pi(\mathrm{xg}) \mathrm{ug}, \mathrm{x} \in \mathrm{l}_{1}(\mathrm{G}, \mathrm{A})
$$

The full cross product of $(A, G, a)$, denoted by $A \rtimes a G=A \rtimes G$ is the completion of $l 1(G, A)$ or $c c(G, A)$ with respect to the norm

$$
\|x\| u=\|(\pi \times u)(x)\|, x \in l_{1}(G, A)
$$

The supremum taken over all (cyclic) covariant representations $(\pi, u, H)$ of $(A, G, a)$. To define the reduced crossed product, we assume that $A \subseteq B(H)$ is faithfully represented and define and we define a faithful representation $\pi: A \rightarrow B(H \otimes I 2(G))$ and a unitary representation $\lambda: G \rightarrow B(H \otimes I 2(G))$ by

$$
\pi(a)(\xi \otimes \delta s)=(s-1 a) \xi \otimes \delta s, \lambda g(\xi \otimes \delta s)=\xi \otimes \delta g s, a \in A, \xi \in H, g s \in G
$$

It is verifiable, $(\pi, \lambda, \mathrm{H} \otimes \mathrm{I} 2(\mathrm{G}))$ is a covariant representation of $(A, G, \alpha)$ call a regular representation of the $c^{*}$-dynamical system. Again, $\lambda$ is actually an amplification of the left regular representation of $G$ on $I_{2}(G)$. The associated form $\pi \times \lambda: I_{1}(G) \rightarrow B\left(H \otimes I_{2}(G)\right)$ is faithful, and the reduced crossed product $A \rtimes a, r G=A \rtimes r G$ is the completion of $I(G, A)$ or $c c(G, A)$ in the reduced norm

$$
\|x\| r=\|(\pi \times \lambda)(x)\| B\left(H \otimes I_{2}(G)\right), x \in l_{1}(G, A)
$$

Equivalently, $A \rtimes r G$ (cf. [25, Chapter 4.1]) can be taken to be the norm closure of the image of $\pi \times \lambda$ or $\pi \times \lambda \mid c c(G, A)$. Clearly, $A \rtimes r G$ does not depend on the choice of faithful representation $A \subseteq B(H)$ (see, e.g., [11, Proposition 4.1.5]). We now define

a G-action on $A \rtimes r G$ by means of the inner automorphisms $g \mapsto A d(\lambda g)$, so that the inclusion $A \subseteq A \rtimes r G$ is $G$-equivariant. Identifying $A$ via its image under $\pi$, then the reduced crossed product also has the nifty property of admitting a faithful conditional expectation $E A: A \rtimes r G \rightarrow A$ that is G-equivariant and uniquely satisfies $E A(x)=x 1$ for all

$$
x=\sum g \in G \times g \lambda g \in l(G, A) \subseteq A \rtimes r G
$$


We referred to the above inclusion as the canonical conditional expectation and write $E$ instead of EA if the dynamical system is clear from the context. The existence of a faithful conditional expectation of $A \rtimes r G$ onto Aalso characterizes the reduced crossed product among $c^{*}$-algebras generated by the image of the integrated form of covariant representation of $(A, G, a)$ [48, Theorem 4.22]. In fact, it holds in more general, if $\mathrm{H} \subseteq \mathrm{G}$ is a subgroup, then there exists an injective *-homomorphism $\mathrm{A} \rtimes r \mathrm{H} \rightarrow \mathrm{A} \rtimes r \mathrm{r}$ that extends the inclusioncc $(H, A) \rightarrow c c(G, A)$. Again, if we identify $A \rtimes r H$ with its image under this * homomorphism, then there exists a faithful conditional expectation $E H: A \rtimes r G \rightarrow A \rtimes r H$ that uniquely satisfy $\mathrm{EH}(\lambda \mathrm{g})=0$, forallg $\notin \mathrm{H}$. We shall proof in the more general case using reduced twisted crossed products in Theorem 3.2 inspired by [3, Theorem 2.2]. In particular, we obtain the generalization of [10, Theorem 7.1] with the aid ofCorollaries 3.3, 3.4, and 3.5 while Lemma 3.6 is the generalization of [10, Theorem 7.2]. Again, Theorem 3.7 is the generalization of [4, Proposition 3.13]. Furthermore, we not that if $A, B$ are $G-c_{i}$-algebras and $\varphi: A \rightarrow B$ is a $G-i$ equivariant c.c.p. map, then the map $\varphi: 11(G, A) \rightarrow \mid 1(G, B)$ given by

$$
\varphi(x) g=\varphi(x g), x \in l_{1}(G, A), g \in G
$$

extends to a c.c.p. map $\sim \varphi: A \rtimes r G \rightarrow B \rtimes r G$. Thus, $\sim \varphi$ uniquely satisfies

$$
\sim \varphi(a \lambda g)=\varphi(a) \lambda g, a \in A, g \in G
$$

It turns that a property of $\varphi$ is inherited by $\sim \varphi$. It is easy to show that this include faithfulness, surjectivity and being a *-homomorphism.

Definition 1.2 For an action of a discrete group $G$ on a topological free space $X$, define $X g=\{x \in X \mid g x=x\}, g \in G$, we say that the action of $G$ on $X$ is topologically free if $X g$ has empty interior for all $g \in G \backslash\{1\}$.

Definition 1.3 LetAand $\mathcal{B}$ be $c^{*}$-algebras and let $\varphi$ be a c.c.p map $\varphi: A \rightarrow B$. The multiplicative domain mult $(\varphi)$ of $\varphi$ is the subset of $A$ given by mult

$$
(\varphi)=\left\{a \in A \mid \varphi\left(a_{i} a\right)=\varphi(a) ; \varphi(a), \varphi\left(a_{i}\right)=\varphi(a) \varphi(a) ;\right\}
$$

From the result of Choi [12, Theorem 3.1],

$$
\operatorname{mult}(\varphi)=\{\mathrm{a} \in \mathrm{A} \mid \varphi(\mathrm{ax})=\varphi(\mathrm{a}) \varphi(\mathrm{x}), \varphi(\mathrm{xa})=\varphi(\mathrm{x}) \varphi(\mathrm{a})\}, \forall \mathrm{x} \in \mathrm{A}
$$

Moreover, if $B \subseteq A$ is a $c^{*}$-algebras and $\varphi: A \rightarrow B$ is a c.c.p map that restricts to the identity map on $\mathcal{B}$, then $\varphi$ is in fact is conditional of $A$ onto $\mathcal{B}$.

Lemma 1.4(Archbold and Spielberge [1, Theorem 1]): LetX be a compact G-space on which the action of $G$ is topologically free. If $I \subseteq C(X) \rtimes r G$ is closed ideal such that $I \cap C(X)=\{0\}$, then $I=\{0\}$.

Following the original article [1], one can easily verify that Lemma 1.4 holds true for topologically free action on $c^{*}$-algebras that are possibly non-unital and noncommutative.If $X$ is compact $\mathrm{G}$-space and $x \in X$, then by composing the faithful conditional expectation, *-homomorphism, Gxo-equivariant *homomorphism $\delta x: C(X) \rightarrow C$, we obtains a u.c.p map 


$$
\begin{gathered}
E G \times 0: C(X) \rtimes r G \rightarrow C(X) \rtimes r G \times 0, \\
C(X) \rtimes r G \times 0 \rightarrow C r i(G \times 0) \\
E x: C(X) \rtimes r G \rightarrow C r \times(G \times 0),
\end{gathered}
$$

Satisfying

$$
\operatorname{Ex}(f \lambda g)=f(x) E G x o(\lambda g), f \in C(X), g \in G
$$

The following result is a reformulation of Kawabe [34]

Theorem 1.5 ([34, Lemma 2.4]): Let X be a compact G-space for which $\{x \in X \mid G x$ oisamenable $\}$ is dense in $X$. If the action of $G$ on $X$ is not topologically free, then there exists a non-zero closed ideal $I \subseteq C(X) \rtimes r G$ for whichIกC $(X)=\{0\}$.

For purpose of clarity, we omit the proof of Theorem 1.5 .

Theorem 1.6(Frolik [22, Theorem 3.1]): Let $X$ be a Stonean space (see Appendix B). If $f: X \rightarrow X$ is homeomorphism, then the fixed point set of $f$ is clopen. In particular, a group action on $X$ is topologically free if and only if it is free. Boundary action are intimately connected with several commutative $C^{*}$-algebras that are of interest in the study of $c^{*}$-simple groups (i.e. groups with simple reduced group $c^{*}$-algebras) can be found in the literatures $[16,18,24,26,31,32,40$, and 42]. The concept of boundary action was originally introduced by Furstenberg [24]. The main idea is to describe to what degree a fixed group of homeomorphism of space (i.e., a fixed non-trivial translation of $\mathrm{R}$ to any bounded subset) can map any or at least some points in the boundary of $R \in R_{\dot{c}}$, namely $(-\infty,+\infty)$ in space. It is clear that any non-trivial translation of $\mathrm{R}$ with positive derivative move any point in $\mathrm{R} U\{\infty\}$ closer to $+\infty$, and that $\{ \pm \infty\}$ are the only fixed point. The study of boundary actions and ideal structure of reduced crossed products have recently be linked to the study of $c^{*}$-simple group (see. [16]). Furthermore, a discrete group can only be $c^{*}$-simple when the $c^{*}$-algebra associated to its regular representation is simple. This property for discrete group pioneered by powers will be one of the focuses of this paper. In particular, Our motivations are the advances in [19, 20, and 21] which were later elaborated in [28]. It is our purpose in this paper to extend the Theorem of Kalanter and Kennedy [32, Theorem 6.2] that characterizes $c$-simplicity in terms of boundary actions to the equivalence of topological freeness due to Kalanter, Kennedy, Breuillard and Ozawa [10, Theorem 3.1] and then generalized some of their results.We remark that other characterization of $c^{*}$-simplicity have been obtained since the result of Kennedy and Kalantar. A few of which we now review;

I. Simplicity of reduced crossed products. Breuillard, Kalantar, Kennedy and Ozawa proved that c*simple discrete groups have the property that

a reduced crossed product $A \rtimes r G$ of a unital G-C*-algebra by $G$ is simple if and only if $A$ is $G$-simple, which means that $A$ has no non-trivial Ginvariant closed ideals [10, Theorem 7.1]. This settled in affirmative a question of de la Harpe and Skandalis [21]. We will generalize this result in section 3. 
II. An averaging property. Haagerup [29] and Kennedy [36] independently proved that a discrete group $G$ is $c^{*}$-simple if and only if for all $t 1, t 2, \ldots, t m \in G \backslash\{1\}$ and $\epsilon>0$ there exists $s 1, \ldots$, sn $\in \mathrm{G}$ such that

$$
\left\|_{1} / n \sum k=1 n \lambda s k t j s k-1\right\|<\epsilon
$$

Clearly, this is an important characterization, because many previously study classes of $c^{*}$-simple groups were always shown to satisfy at most minor variant of the latter property. In fact, it is nonetheless part of the original proof of powers that F2 is $\mathrm{c}^{*}$-simple. We prove in section 3 that the reduced crossed products over $c^{*}$-simple groups satisfy a similar property. We also record that the above property is a group $C^{*}$-algebra variant of the Dixmier property. A unital $c^{*}$-algebra $A$ is said to satisfy the Dixmier property if the closed convex hull of $\left\{u_{u} u_{i} l u \in U(A)\right\}$ intersects the centre of $A$ for all $a \in A$. Haagerup and Zsidó [30] proved that a unital, simple c*-algebraA always satisfies the Dixmier property, and that the intersection of the aforementioned closed convex hull and the centre always reduces to a point, if $\mathrm{c}^{*}$-algebra has a unique tracial state [30].

III. Recurrent subgroups. Independently, Kennedy [36] obtained an algebraic characterization of $c *$ simplicity using the notion of recurrence for subgroups, hence a group-theoretical version of the topological dynamical notion of uniformly recurrent subgroup. A subgroup $\mathrm{H}$ of a group $\mathrm{G}$ is recurrent if there exists a final subset $F \subseteq G \backslash\{1\}$ such that

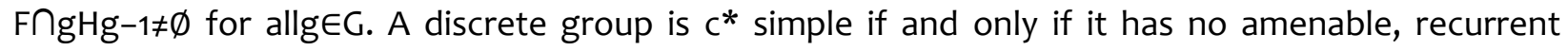
subgroups.

Theorem 1.7(Breuillard, Kalantar, Kennedy and Ozawa [10, Corollary 4.3]): Let $G$ be a discrete group with amenable radical $R(G)$. Then $g \in G$ satisfies $\tau(\lambda g)=0$ for all tracial states $\tau$ on $\mathrm{Cr} i(G)$ if and only if $g \notin R(G)$. In particular, $G$ has the unique trace property if and only if $R(G)=\{1\}$

The proof of the implication of the infamous result (Theorem 1.7) requires generalization. We differ this until section 3 (Theorem 3.8). However, Theorem 1.7 partially settles the question of de la Harpe; whether there exist $c^{*}$-simple groups without the unique trace property. Conversely, by composing the conditional expectation $\mathrm{Cr} i(G) \rightarrow \mathrm{Cr} i(R(G)$ ) with trivial representation $\mathrm{Cr} i(R(G)) \rightarrow C$ (i.e., an existence result which follows from the amenability of $R(G)$ [11, Theorem 2.6.8]), yields a state $\tau: C r$ $i(G) \rightarrow C$ such that $\tau(\lambda g)=1$ for all $g \in R(G)$. Since $R(G)$ is normal, then for any two $g$, heG we have ghe $R(G)$ if and only if $h g \in R(G)$, implying $\tau(\lambda g \lambda h)=\tau(\lambda h \lambda g)$. Hence $\tau$ is a tracial state on $\mathrm{Cr}_{i}(R(G))$. The rest of this paper is organized as follows: In section 2, we give some preliminary results which we shall need later. In section 3, we proof our main results. Precisely, we proof Theorem 3.1, 3.2, 3.7, and 3.8. In section 4, we study stability properties that our results and many others in the literatures satisfy. Specifically, we give some examples of what stability properties that classes of c*simple groups and groups with trivial amenable radical satisfy. Furthermore, we establish stability criteriato ensure that a lot of other stability properties are automatically satisfied for any class of groups. Finally, we give in section 5 , some examples of $c^{*}$-simple groups, mainly using the characterization of $c^{*}$-simplicity arising from Theorem 3.1. 
2. PRELIMINARIES We shall need the following Lemmas. We prove Lemma 2.11 for the sake of completeness.

Lemma 2.1 ([27, see also 24, Lemma 4.1]): Let $G$ be a Hausdorff topological group and let X be a minimal, proximal compact $\mathrm{G}$-space. If $\mathrm{X}$ has an isolated point, then $\mathrm{X}$ is a one-point space.

Lemma 2.2 ([28]): Let $X$ be a minimal compact G-space. If the action of $G$ on $X$ is proximal, then the only G-equivariant continuous map is the identity map.

Lemma 2.3 ([28]): Let $G$ be a topological group, let (Xi)iel be a family of compact $G$-spaces and let $X=\prod i \in I X i$ be the product space equipped with the diagonal Gaction. Then the action of $G$ on $X$ is proximal (resp. strongly proximal) for all $i \in I$.

Lemma 2.4 ([20]): Let $G$ be a non-elementary hyperbolic group. Then the action of $G$ on itself by left translation induces a boundary action of $\mathrm{G}$ ondG.

Lemma 2.5 ([37, proposition 3.1, see also 38, Proposition 4.26]): Let T be a countable, leafless tree and let $G$ be a discrete group acting minimally on $T$ by automorphisms without inversion. If the action $G$ on $\partial \mathrm{T}$ is non-elementary, then ' $\partial \mathrm{T}$ is a G-boundary in the shadow topology.

Lemma 2.6 ([24]): Let $G$ be a Hausdorff topological group. $X^{\prime}$ is a compact minimal G-space and $X$ is a G-boundary, then there is at most one G-equivariant u.c.p map $C(X) \rightarrow C(X)$ and it is an injective *homomorphism.

Lemma 2.7 ([32, see also 10, Proposition 2.5]): Let $G$ be a Hausdorff topological map and let $A$ be a unital G-invariant $C^{*}$-subalgebra of a unitalG-C*-algebraB. Then any $G$-equivariant u.c.p map $A \rightarrow C(\partial F G)$ extends to a G-equivariant u.c.p mapB $\rightarrow C(\partial F G)$.

Lemma 2.8 ([15, 1(1957), pp. 509 - 544]):LetN be a closed, normal, amenable subgroup of a locally compact group $\mathrm{G}$ and let $\mathrm{X}$ be a G-bonudary. Then $\mathrm{N}$ acts trivially on $\mathrm{X}$.

Lemma 2.9 ([23, Proposition 7]): Let $G$ to be a locally compact group. Then $R(G)=\cap x \in \partial F G G x$. In particular, $R(G)=\{1\}$ if and only if $G$ admits a faithful boundary action. Moreover, $\partial F G$ is G-equivariantly homeomorphic to $\partial F(G / R(G))$.

Lemma 2.10 ([27]): For any discrete group $G$ and any $x \in \partial F G$, the stabilizer $G x$ is an amenable subgroup of $G$.

Lemma 2.11 (Special case of [1, Theorem 1]): Let $(A, G, \alpha, \beta)$ be a unital twisted $c$ dynamical system and let $X$ be the maximal ideal space of the centre $Z(A)$ of $A$. Assume that the action of $G$ on $X$ is free. If $J$ is a closed ideal in $A \rtimes a, r \beta G$, then for $J A=J \cap A$ we have $J A \rtimes a, r \beta G \subseteq J A^{\prime} \rtimes a, r \beta G$.

Proof: Let $I A=I \cap A$ and let $\pi: A \rightarrow A / I A$ be the quotient map. We assume that $\rho: A / I A \rightarrow B(H)$ is an irreducible representation of $A / I A$. Now, consider the representation $A+I \rightarrow(A+I) / I \cong A / I A \rho \rightarrow B(H)$. $B y$ Arveson's extension theorem, this map extends to a u.c.p map $\varphi: A \rtimes \alpha, r \beta G \rightarrow B(H)$ such that $\varphi(I)=0$ and $A \subseteq$ mult $(\varphi)$, since $\varphi \mid A=\rho \circ \pi$. By irreducibility, the restriction of $\varphi$ to $Z(A) \cong C(X)$ is a point mass on 
$X$, i.e., $\varphi \mid Z(A)=\delta x$ for some $x \in X$. Let $g \in G \backslash\{1\}$, then there exists $f \in C(X)$ such that $f(g-1 X) \neq f(x)$. This implies $\varphi(\lambda \beta(g)(f \quad(x)) 1 H)=\varphi(\lambda \beta(g) f)=\varphi(g f \quad \lambda \beta(g))=f \quad(g-1 x) \varphi(\lambda \beta(g))$ Therefore, $\varphi(\lambda \beta(g))=0$. Let $E A: A \rtimes a, r \beta G \rightarrow A$ be the canonical conditional expectation, it follows that $\varphi=\varphi \circ E A$.

Hence,

$$
\rho(\pi(E A(I)))=\varphi(E A(I))=\varphi(I)=\{0\}
$$

Since $\rho$ was arbitrary, $\pi(E A(I))=\{0\}$, so that $E A(I) \subseteq I$. For any positive element $x \in I$, let $\ell$ be the image of $x$ under $\pi: A \rtimes a, r \beta G \rightarrow(A / I A) \rtimes a, r \beta G$, let $E A / I:(A / I A) \rtimes^{\star} \alpha, r^{*} \beta G \rightarrow A / I A$ be the canonical faithful conditional expectation. SinceEA/lo $\pi=\pi \circ E A$, it follows that $E A / I(I)=0$ since $E A(x) \in I \cap A$. Since $E A / I$ is faithful, $\ell=0$ and $x \in I A{ }^{\prime} \rtimes \alpha, r \beta G$. $\square$ Moreover, fore any $(A, G, \alpha, \beta)$, twisted $c^{*}$-dynamical system and any G-invariant, closed ideal $I \in A^{\prime} \rtimes a, r \beta G$, the commutative diagram o $I \rtimes a, r \beta G A \rtimes a, r \beta G A / l \rtimes{ }^{*} \alpha, r^{\prime} \beta G \sim$ $\pi 0$

o I A $\pi A / I O$

arise when $\pi$ induces a surjective ${ }^{*}$-homomorphism $\sim \pi$ :Aメa,r $\beta G \rightarrow A / I \rtimes^{*} \alpha, r^{*} \beta G$ at the level of crossed products yields the identity $\left(I^{\prime} \rtimes a, r \beta G\right) \cap A=I$ (2.2) Thus, El,EAandEA/I denote the canonical conditional expectation respectively. Furthermore, for any G-boundary X.If $A$ is unital, then for the natural extension $(A \otimes C(X), G, \mu, \gamma)$, we found that if $K \subseteq(A \otimes C(X)) \rtimes \mu, r \gamma G$ is a closed ideal and $K A=K \cap(A \otimes C(X))$, then there is a commutative diagram of *-homomorphisms

$$
\begin{gathered}
A \rtimes a, r \beta G \quad(A \otimes C(X)) \rtimes \mu, r \gamma G \\
A /(K \cap A) \rtimes \cdot a, r \cdot \beta G \quad(A \otimes C(X)) / K A \rtimes \mu, r \gamma G
\end{gathered}
$$

where the horizontal arrows are injective. It follows that

$$
\left(K A^{\prime} \rtimes \mu, r \gamma G\right) \cap(A \rtimes a, r \beta G)=(K \cap A)^{\prime} \rtimes a, r \beta G
$$

\section{MAIN RESULTS}

We now prove the following

Theorem 3.1 (Main Theorem): Let $G$ be a discrete group. Then the followingare equivalent,simple (I IV), and topologically free (V - VI).
I. $G$
II. $\quad C(X) \rtimes r G$, for some G-boundary $X$
III. $\quad C(X) \rtimes r G$, for all G-boundary $X$
IV. $\quad C(\partial F G) \rtimes r G$
V. The action of $\mathrm{C}$ on some $\mathrm{X}$
VI. The action of $G$ on $\partial F G$ 
Proof: Clearly, III implies II, III imply IV and IV implies V are trivial. Now, by Theorem 1.6, the action of G on $\partial F G$ is topologically free if and only if it is free, since $\partial F G$ is Stonean (see, [14, Theorem 3.1]). Again V imply II, VI imply IV follow from Lemma 1.4. If C $(\partial F G) \rtimes r G$ is simple, then all stabilizer subgroups for the Gaction on $\partial F G$ are amenable by Lemma 2.10. The action of $G$ on $\partial F G$ is topologically free by Theorem 1.5, thus proving IV implies VI. Next, we need to prove I imply III. Let X be a G-boundary, using Lemma 2.6, we may assume that there is a G-equivariant unital $c^{*}$-algebra inclusion $C(X) \subseteq C(\partial F G)$. Let $\pi: C(\partial F G) \rtimes r G \rightarrow B$ be a unital *-homeomorphism. The action of $G$ on $\mathcal{B}$ may be defined by means of inner automorphisms $\mathrm{Ad}(\pi(\lambda \mathrm{g}))$ of $\mathcal{B}$, so that $\pi$ becomes Gequivariant. Using the inclusion $C \subseteq C(X)$, we realizeCr $C_{i}(G)$ as a unitalG-invariant $c^{*}$ subalgebra of $C_{i}(X) \rtimes r G$. If $\mathrm{Cr}_{i}(G)$ is simple, then $\pi \mid C r i(G)$ is injective, so that canonical tracial state of $\tau: \mathrm{Cr} i(G) \rightarrow C \subseteq C(\partial F G)$ extend to G-equivariant u.c.p map $\tau: B \rightarrow C(\partial F G)$ such that $\sim \tau \circ \pi \mid C r i(G)=\tau$ by Lemma 2.7. Using Lemma 2.6 once again, we find that the map $\sim \tau \circ \pi \mid C(X): C(X) \rightarrow C(\partial F G)$ is the inclusionmap $C(X) \rightarrow C(\partial F G)$. Precisely, $C(X) \subseteq$ mult $(\sim \tau \circ \pi)$. If $E: C(X) \rtimes r G \rightarrow C(X)$ is the canonical faithful conditional expectation, the $\sim \tau(\pi(f \lambda g))=f \tau(\lambda g)=E(f \lambda g)$ in $C(\partial F G)$ for all $f \in C(X)$ and $g \in G$. Hence, $\sim \tau \circ \pi=E$, meaning that $\pi$ is faithful and therefore injective. Henceforth, $C(X) \rtimes r G$ is simple.Next, we need to prove II implies I. If $C(X) \rtimes r G$ is simple for some $G$ boundary $X$, let $I \subseteq C r i(G)$ by a proper closed ideal. If $\varphi: C r i(G) \rightarrow C$ is a state such that $\varphi(I)=\{0\}$ extend $\varphi$ to a state on $C(X) \rtimes r G$ and let (gi) be a net in $G$ such that gi $\mu \rightarrow \delta x$ for some $x \in X$ where $\mu=\varphi \mid C(X)$. By weak $^{*}$ - compactness we may assume that $(\varphi \circ A d(\lambda g))$ converges to some state $\psi$ on $C(X) \rtimes r G$, so that $\psi \mid C(X)=\delta x$ and $\psi \mid I=0$. Thus, $C(X) \subseteq$ mult $(\psi)$.Furthermore, for any $b \in I, f 1 f 2 \in C(\partial F G)$ andf $1 f 2 \in G$,

$$
\psi\left(f_{1} \lambda g_{1}\right) b\left(f_{2} \lambda g_{2}\right)=f_{1}(x) \psi\left(\lambda g 1 b \lambda g_{2}\right) f_{2}(g 2 x)=0, \lambda g 1 b \lambda g 2 \in I
$$

It is not difficult to see that the ideal generated by $I$ is proper. Therefore we have $I=\{0\}$ because $C(\partial F G) \rtimes r G$ was assumed to be simple.

Remark 3.2: It follows from Theorem 3.1 that any $c *$-simple discrete group $G$ has trivial amenable radical. Indeed, if the action of $G$ on $\partial F G$ is free, then

$R(G)=\bigcap x \in \partial F G G x=\{1\}$ by Lemma 2.9. Since the result of Kalantar and Kennedy [90], other characterizations of $c^{*}$-simplicity have been obtained (see, [4, 10, 29, 30, and 36]). Moreover, some of these results in the later literatures required generalization. Precisely, [10, Theorem 7.1, 7.2, Corollary 4.3], [36, Definition 5.2] and [4, Proposition 3.13].

Assume that $(A, G, a)$ is separable. If $G$ is amenable, then every primitive ideal of $A \rtimes r G$ is an induced primitive ideal. Moreover, if $G$ acts freely on $\operatorname{prim}(A)$, then the induce process establishes a bijection between prim(AメrG) and the quasiorbits in prim(A). In particular, if $G$ acts freely and every orbit is dense, then $A \rtimes r G$ is simple. It is instructive to note that the twisted action and the equivalence of a group being $c^{*}$-simple admits a free boundary action allows us to generalize many of these results. This we do in the following theorems.

Theorem 3.2: Let $(A, G, a, \beta)$ be a unital twisted $c^{*}$-dynamical system where $G$ is $c^{*}$ simple. For a maximal ideal I of $A \rtimes a, r \beta G, I \cap A$ is a maximal G-invariant ideal of $A$. Conversely, for a maximal G-invariant ideal $\gamma$ of $A$, the ideal $\gamma^{\prime} \rtimes a, r \beta G$ of $A \rtimes a, r \beta G$ is maximal. Moreover, the correspondence is bijective. 
Proof: Let $\gamma$ be a maximal G-invariant ideal in $A$. We claim that the ideal $\gamma^{\prime} \rtimes a, r \beta G$ in $A^{\prime} \rtimes a, r \beta G$ is maximal; assume thatJ is a proper ideal in $A{ }^{\prime} \rtimes a, r \beta G$ such that $\gamma^{\prime} \rtimes a, r \beta G \subseteq J$. Now, let $(A \otimes C(\partial F G), G, \nu, l)$ of $(A, G, \alpha, \beta)$ be the natural extension.

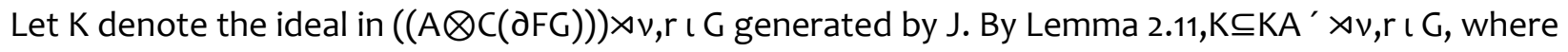

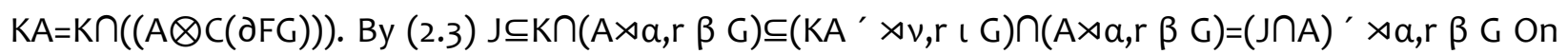

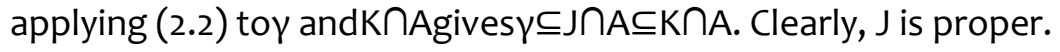

Theorem 3.6 implies that $K$ is proper, so the maximality of $\gamma$ implies that $\gamma=K \cap A$ since $K \cap A$ is a $G$ invariant. It follows that $J \subseteq \gamma^{\prime} \rtimes a, r \beta G$, and $\gamma^{\prime} \rtimes a, r \beta G$ is maximal. We required an analysis to show that the ideal I $\cap A$ is maximal among proper Ginvariant ideals inA. Now let I be a maximal ideal inA ' $\rtimes a, r \beta G$. LetJ denote the ideals in

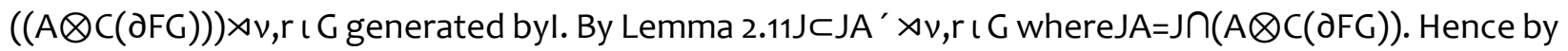

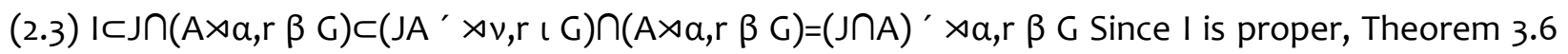
implies that $J \cap A$ is proper inA, whence maximality of I implies that $I=(J \cap A)^{\prime} \rtimes a, r \beta G$. Now $I \cap A=J \cap A$ follows from (2.2). It follows from our analysis $I=(I \cap A)^{\prime} \rtimes a, r \beta G(3.1)$ Now, let $F$ be a proper $G$-invariant

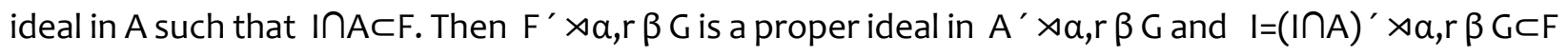
$\rtimes a, r \beta G$.

Therefore the maximality of I implies that $I=F^{\prime} \rtimes a, r \beta G$. Hencel $\cap A=\left(F^{\prime} \rtimes a, r \beta G\right) \cap A=F$. Thus, InA is maximal. Finally, it now clear the correspondence is bijective follows from the identities (2.2) and (3.1)

Corollary 3.3:Let $(A, G, \alpha, \beta)$ be a unital twisted $C^{*}$-dynamical system where $G$ is $C^{*}$-simple. Then $A \rtimes a, r$ $\beta G$ is simple if and only if $A$ is $G$-simple.

Corollary 3.4: If $G$ is $C^{*}$-simple, then the reduced twisted group $C^{*}$-algebra $\mathrm{Cr}_{c}(G, \beta)$ is simple for every multiplier $\beta: G \times G \rightarrow \mathbb{\Gamma}$.

Corollary 3.5:Let $(A, G, \alpha, \beta)$ be as in Corollary 3.3. Let $N$ be a normal subgroup of $G$. Write $(\alpha, \beta)$ for the restriction of $(\alpha, \beta)$ to $N$. If $G / N$ is $C^{*}$-simple, then $A \rtimes \alpha, r \beta G$ is simple whenever $A \rtimes \alpha, r \beta$ Nis simple.

Proof: $A \rtimes a, r \beta G \cong(A \rtimes a, r \beta N) \rtimes v, r$ । $(G / N)$ follow from the existence of a twisted action $(l, v)$ of $\mathrm{G} /$ NonA $\rtimes a, r \beta \mathrm{N}$. The desire conclusion now follows from Corollary 3.3.

Remark 3.6: Corollary 3.3 and Corollary 3.4 gives the generalization of [10, Theorem 7.1]. It should be noted that the conclusion of Theorem 3.2 is not true if we allow the underlying $c$-algebra to be nonunital. Thurs, $\operatorname{co}(X)$ is always $G$ simple, even though $\operatorname{co}(X) \rtimes r G$ may contain many ideals. Furthermore, assume that $G$ is a $C^{*}$-simple group and $A$, a unital $G$ - $C^{*}$-algebra. If $Z(I(A))$ is $G$-simple, and $A$ is prime, then the action of $G$ on $A$ has the intersection property

(i.e., Z(I(A)) $\rtimes r G$ is simple, see., [34, Theorem 3.4]), and A $\rtimes r G$ is prime respectively. Indeed, there is an injective map of the set of prime and G-invariant ideals to the set of prime ideals in $A \rtimes r G$, given by $\mid \mapsto I$ ' $\rtimes r G$. In fact, if $I \subseteq A$ is a prime, and G-ivariant ideal, then $A / I$ is prime $C^{*}$-algebra and $I(A) \rtimes r G$ is a prime 
$C^{*}$-algebra by our analysis above. Thus, I' $\rtimes r G$ is a prime ideal of $A \rtimes r G$, therefore the map $I \mapsto I^{\prime} \rtimes r G$ is

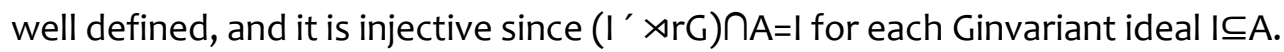

Theorem 3.7:Let $(A, G, a, \beta)$ be a unital twisted $C^{*}$-dynamical system. Let $X$ be a $G$-boundary and let $(A \otimes C(X), G, v, l)$ denote the associated natural extension. Let I be a proper ideal in

$A \rtimes a, r \beta G$ and let $J$ denote the ideal in $(A \otimes C(X)) \rtimes \nu, r$ I G generated by I. Then J is proper.

Proof: Let $\varphi$ be a state on $A \rtimes a, r \beta G$ such that $\varphi(I)=0$. By $[41,48]$ there is a state $\psi$ on $(A \otimes C(X)) \rtimes v, r$ ı $G$, a net (gi) $\in G$ and $x \in X$ such that $\psi \mid A \rtimes a, r \beta G=\lim _{c} j \psi \circ A d(\lambda \beta(g j)) ;$ and $\psi \mid C(X)=\delta x$. It should be noted that $\psi \mid A \rtimes a, r \beta C(I)=0$, and $C(X) \in \psi$. Hence for $b \in I, a 1, a 2 \in A, d 1, d 2 \in C(X)$ and $\varsigma 1, \varsigma 2 \in G$ we have

$$
\begin{gathered}
\psi\left((\mathrm{a} 1 \otimes \mathrm{d} 1) \lambda \mathrm{l}\left(\mathrm{s}_{1}\right) \mathrm{b}(\mathrm{a} 2 \otimes \mathrm{d} 2) \lambda \mathrm{l}\left(\varsigma_{2}\right)\right) \\
\dot{\mathrm{d} 1}(\mathrm{x}) \psi(\mathrm{a} 1 \lambda \beta(\varsigma 1) \mathrm{ba} 1 \lambda \beta(\varsigma 2)) \mathrm{d} 2(\mathrm{~d} 2 \mathrm{x})
\end{gathered}
$$

¿0

It follows that $\psi(J)=0$. Hence $J$ is proper.

Remark 3.8: Theorem 3.7 above generalizes [10, Lemma 7.2].

\section{STABILITY PROPERTIES}

Here we establish stability criteria to ensure that many stability properties are automatically satisfied for any class of groups.

Theorem 4.1:Let $\Gamma$ be a property for discrete groups such that

T 1. The trivial group $\{1\}$ has property $\Gamma$

T 2. If $G$ has property $\Gamma$, then $G$ is ice

T 3. If $\mathrm{N}$ is normal subgroup of $\mathrm{G}$, then $\mathrm{G}$ has property $\Gamma$ if and only if $\mathrm{CC}(\mathrm{N})$ have property $\Gamma$

Then the following hold:

L 1. $G_{1} \times G_{2}$ has property $\Gamma$ if and only if $G_{1}$ and $G_{2}$ has property $\Gamma$

L 2. $G$ has property $\Gamma$ if and only if $\operatorname{Aut}(G)$ has property $\Gamma$

L 3. If $\mathrm{N}$ is normal subgroup of $\mathrm{G}$ such that $\mathrm{N}$ and $\mathrm{G} / \mathrm{N}$ have property $\Gamma$, then $\mathrm{G}$ has property $\Gamma$

L 4. If $H$ is a finite index subgroup of $G$, then $G$ has property $\Gamma$ if and only if $G$ is ice and $H$ has property Г. Proof:

L1 is clear from T3. For L2, one can identify copy of $G$ with the normal subgroup of Aut( $G)$ of linear automorphisms, since $G$ is ice by $T 2$ and $T 3$. As $C A u t(G)(G)=\{1\}$, L2 follows immediately. For $L 3$, we need to show that $C G(N)$ has property $P$. Since $N C G(N)$ and $N C G(N) / N$ are normal in $G$ and $G / N$ respectively, hence $C G(N)$ has property $P$. Because $N$ is centerless and ice due to $T 2, N C G(N) / N$ is isomorphic to 
$\mathrm{CG}(\mathrm{N}) / \mathrm{N} \cap \mathrm{CG}(\mathrm{N})=\mathrm{CG}(\mathrm{N})$. For $\mathrm{L} 4$, one can assume $\mathrm{G}$ is ice by $\mathrm{T} 2$, and let $\mathrm{N}=\mathrm{\cap g} \in \mathrm{GgHg}-1$. Then $\mathrm{N}$ is the kernel of the canonical action of $\mathrm{G}$ on the final coset space $\mathrm{G} / \mathrm{H}$ sometimes called the normal core of $\mathrm{H} \in \mathrm{G}$, so it is a normal finite-index subgroup of $\mathrm{G}$. It follows from our analysis that any element $x \in C G(N)$ has finite conjugacy class in $G$, and $C G(N)=\{1\}$. Since $N, H, G$ has property $P$, then $C H(N) \subseteq C G(N)=\{1\}$.

Remark 4.2: It is instructive to note that $\mathrm{G}$ could be isomorphic to the direct product of $\mathrm{H}$ and a finite cyclic group. Thus, $\mathrm{G \subset H}$ is not necessary ice. As immediate consequence of theorem 4.1, we have the following proposition;

Proposition 4.3: Let $\mathrm{G}$ be a discrete group with a normal subgroup $\mathrm{N}$. Then $\mathrm{G}$ has trivial amenable radical if and only if $\mathrm{N}$ and $\mathrm{CG}(\mathrm{N})$ has trivial amenable radical.

It is clear, the amenable radical is characteristic, i.e., $\alpha(H)=H$ for any automorphisms $\alpha \in A u t(H)$. If $H \subset C$ is a normal subgroup, the conjugation by $g \in G$ is an automorphisms of $H$, implying $g R(H) g-1=R(H)$. Therefore, $R(H)$ is normal in $G$ and amenable, so that $R(H) \subseteq R(G) \cap H$. Thus, $R(G) \cap H$ is amenable and normal in $H, R(H)=R(G) \cap H$. We now prove the claim. Since $N$ and $C G(N)$ are normal, $R(G)=\{1\}$ implies $R(N)=R(C G(N))=\{1\}$ by our analysis. Conversely, assume that $R(N)=R(C G(N))=\{1\}$. Then $R(G) \cap N=R(N)=\{1\}$, so normality of $R(G)$ and $N$ implies $g(n g-1 n-1)=($ gng -1$) n-1 \in R(G) \cap N=\{1\}$ for all $g \in R(G)$ and $n \in N$. Therefore, $R(G)$ and $N$ commute, meaning that

$$
R(G)=R(G) \cap C G(N)=R(C G(N))=\{1\}
$$

\section{SOME EXAMPLES OF C*-DISCRETE GROUPS}

We shall need the following Lemma.

Lemma 5.1: Let $G$ be a Hausdorff topological group and let $X$ be a minimal proximal compact $G$-space. If $X$ has an isolated point, then $X$ is a one point space. In all of the following examples, we have assume $X$ to be boundaries that are not one-point spaces, such that $X$ has no isolated points by Lemma 5.1. Precisely, finite subsets of $X$ have empty interior.

Example I (Powers [42]):Non-abelian free groups of finite rank. For $n \geq 2$, the action of non-abelian free group Fn on its boundary $\partial F n$ of one-sided reduced infinite words is topological free. This implies that $\mathrm{Fn}$ is $C^{*}$-simple. Indeed, if $A$ is a free generating set for $\mathrm{Fn}$, let $\Pi=g 1 . . . g n \in F n /\{1\}$ be a word in a reduced form, where g1...gn $\in A \cup A-1$. We claim that $X \Pi$ is finite, so that it has empty interior. Taking the conjugation if necessary, assuming that g1gn $\neq 1$ since $\Pi \neq 1$, then

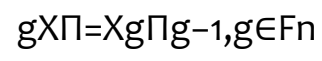

so that $Х \Pi$ has an empty interior if and only if $\mathrm{Xg} \Pi g-1$ has empty interior. If $\Pi x=x$ for some $x \in \partial F n$, assume that the concatenation is reduced. Then the first $n$ letter of $x$ are the $n$ letters of $\Pi$. Since g1gn $\neq 1, \Pi 2 x$ is also reduced. Therefore,

then next $n$ letters of $x$ are those of $\Pi$. On iterating this process, we see that $x=\Pi \Pi \Pi \ldots$, if $\Pi x$ is not reduced, then let $1 \leq k \leq n$ be the largest such that the first $k$ letters of $x$, are gn $-1 \ldots g n-k+1-1$. Since the first letter of $x$ is gn -1 and the first letter of $\Pi x$ is g1, assuming that $k<n$, we have $k=n$. Thus, 
the first $n$ letters of $\Pi-1$. From our analysis so far, we conclude that $x=\Pi-1 \Pi-1 \Pi-1 .$. , finally, $X \Pi$ consists of two points. Hence $\mathrm{Fn}$ is $\mathrm{C}^{*}$-simple.

Example II ([6]):Projective Special linear Groups. For $n \geq 2$, the action of PSL(n,R) on real projective $n-1$ space $\Omega=P n-1(R)$ is topologically free.We need to realize this. Let $\Pi:(R n) o \rightarrow \Omega$ be the quotient map and $g \in S L(n, R)$ fixes a non-empty open subset $U \subseteq P n-1(R)$ pointwise. Let $V \subseteq \Pi-1(U)$ be non-empty open ball in Rn. For $\nu, \varkappa \in V$ such that $\Pi(\nu) \neq \Pi(\varkappa)$ and $\gamma, \gamma^{\prime} \in R$ such that $g \nu=\gamma \nu$ and $g \varkappa=\gamma^{\prime} \varkappa$, then by convexity there exists $\gamma^{\prime \prime} \in \mathrm{R}$ such that

$$
\gamma^{\prime \prime}(\nu+x)=g(\nu+x)=\gamma \nu+\gamma^{\prime} x
$$

Since $\nu \notin R \mathcal{R}$, if follows, $\gamma=\gamma^{\prime}=\gamma^{\prime \prime}$. Therefore $g=\gamma_{1}$ on $V \cup\{0\}$. Assuming $a \in V$, then for all $b \in R n$ there exists $c \neq 0$ such that $c b+a \in V$. By linearity, $g b=\gamma b$. Since $g$ factors to the identity element in $G$, it holds for any discrete subgroup $\Phi \subseteq G$ for which $\Omega$ is a $\Phi$-boundary is $C^{*}$-simple. Precisely, $\operatorname{PSL}(n, Z)$ is $C^{*}$ simple. Many other examples can be found in [17, 43], [10, Section 6.2], [32, Theorem 6.5], [25, Proposition 12], [3, P. 536], [39, Proposition 7.2] and [19, Theorem 2.6] etc.

\section{REFERENCES}

1. R. J. ARCHBOLD AND J. S. SPIELBERG, Topologically free actions and ideals in discrete C*dynamical systems, Proceedings of Edinburg Mathematical Society, 37 (1992), pp. 119 - 124

2. E. BÉDOS, Operator algebras associated with free products of group with amalgamation, MathematischeAnnalen, 266 (1984), pp. $279-286$

3. E. BÉDOS, Discrete groups and simple $C^{*}$-algebras, Mathematical Proceedings of the Cambridge Philosophical Society, 109 (1991), pp. $521-537$

4. E. BÉDOS AND R. CONTI, On maximal ideals in certain reduced twisted C*crossed products, Mathematical Proceedings of the Cambridge Philosophical Society, 158 (2015), pp. 399 417

5. E. BÉDOS AND T. OMLAND, On twisted group $C^{*}$-algebras associated with FChypercentral groups and other related groups, Ergodic Theory and Dynamical Systems, 36 (2016), pp. $1743-1756$

6. B. BEKKA, M. G. COWLING, AND P. DE LA HARPE, Simplicity of the reduced C*-algebra of PSL(n,Z), International Mathematics Research Notices, 7 (1994), pp. 285 - 291

7. B. BEKKA, M. G. COWLING, AND P. DE LA HARPE, Some groups whose rduced $C^{*}$-algebra is simple, Publications Mathématiques de I'THÉS, 80 (1994), pp. 117 - 134

8. M. B. BEKKA AND P. DE LA HARPE, Groups with simple reduced $C^{*}$-algebras, ExpositionesMathematicae, 18 (200), pp. 215 - 230

9. F. BOCA AND V. NITICĂ, Combinatorial properties of groups and simple C*algebras with a unique trace, Journal of Operator Theory, 20 (1988), pp. $183-196$

10. E. BREUILLARD, M. KENNEDY, M. KALANTAR, AND N. OZAWA, C*-simplicity and the unique trace property for the discrete groups, 2016, arXiv: 1410.2518

11. N. P. BROWN AND N. OZAWA, C*-algebras and Finite Dimensional Approximations, vol. 88 graduate Studies in mathematics, American Mathematical Society, 2008 
12. M. D. CHOI, ASchwarz inequality for positive linear maps on $\mathrm{C}^{*}$-algebras, Illinois Journal of Mathematics, 18 (1974), pp. $565-574$

13. M. D. CHOI, A simple $C^{*}$-algebra generated by two finite-order unitaries, Canadian journal of mathematics, 31 (1979), pp. $867-880$

14. M. D. CHOI AND E. G. EFFROS, Injectivity and operator spaces, Journal of functional analysis, 24 (1977), pp. $156-209$

15. M. M. DAY, Amenable semigroups, Illinois Journal of Mathematics, 1 (1957), pp. $509-544$

16. P. DE LA HARPE, Reduced $C^{*}$-algebras of discrete groups which are simple with unique trace, Springer Lecture notes in Mathematics, 1132 (1985), pp. $230-253$

17. $P$. DE LA HARPE, Groupeshyperboliques, algèbres $d$ ' opèrateurs et un thèorème de Jolissaint, CompteaRendus des Sèances de l'Acadèmie des Sciences. Sèrie I Mathématique, 307 (1988), pp. $771-774$

18. P. DE LA HARPE, On simplicity of reduced $C^{*}$-algebra of groups, Bulletin of the London Mathematical Society 39 (2007), pp. $1-26$

19. P. DE LA HARPE AND M. BRIDSON, Mapping Class groups and outer automorphism groups of free groups are $C^{*}$-simple, Journal of Functional Analysis, 212 (2004), pp. 195- 205

20. P. DE LA HARPE, AND J. P. PRÉAUX, C*-simple groups: almagamated free products, hnn extensions, and fundamental group of 3 - manifolds, Journal of Topology and Analysis, 3 (2011), pp. $451-489$

21. P. DE LA HARPE, AND G. SKANDALIS, Powers' and simple C*algebras, MathematischeAnnalen, 273 (1986), pp. $241-250$

22. Z. FROLIK, Maps of externally disconnected spaces, theory of type, and applications, in General Ttopology and its Relations to Modern Analysis and Algebra, Academia Publishing House of the Czechoslovak Academy of Science, 1971, pp. 131 - 142

23. A. FURMAN, On minimal strogly proximal action of locally compact groups, Isreal Journal of Mathematics, 136 (2003), pp. $173-187$

24. H. FURSTENBERG, Boundary theory and stochastic processes on homogeneous spaces, in Harmonic Analysis on Homogeneous spaces, vol. Proceeding of Symposia in Pure Mathematics, 1973

25. T. GIORDANO AND P. DE LA HARPE, Groupes de tresses et moyennabilité intérieure, Arkiv for Matematik, 29 (1991), pp. $63-72$

26. S. GLASNER, Topological dynamics and group theory, Transactions of American Mathematical Society, 187 (1974), pp. $327-334$

27. S. GLASNER, Compressibility properties in topological dynamics, American Journal of Mathematics, 97 (1975), pp. $148-171$

28. S. GLASNER, Proximal Flows, vol. 517 of Lecture Notes in Mathematics, Springer-Verlag, 1976

29. U. HAAGERUP, A new look at C*-simplicity and the unique trace property of a group, 2015, arXiv: 1509.5880

30. U. HAAGERUP AND L. ZSIDÓ, Sur la propriété de Dixmierpour les C*algebres, ComptesRendus de l’Acad'emie des Sciences, Série I, 298 (1984), pp. 173 - 176 
31. M. HAMANA, Injective envelopes of $C^{*}$-algebras, Journal of the Mathematical Society of Japan, 31 (1979), pp. $181-197$

32. M. KALANTAR AND M. KENNEDY, Boundaries of reduced $C^{*}$-algebras of discrete groups, Journal für die Reine und AngewandteMathematik, 727 (2017), pp. 247 - 267

33. I. KAPLANSKY, Functional Analysis, in some aspects of analysis and probability vol. 4 of Surveys in Applied Mathematics, John Wiley \& Sons, Inc, New York, 1958, pp. 1 - 34

34. T. KAWABE, Uniformly recurrent subgroups and the ideal structure of reduced crossed products, 2017, arXiv: 1701.03413

35. S. KAWAMURA AND J. TOMIYAMA, Properties of Topological Dynamical Systems and Corresponding C*-Algebras, Tokoyo Journal of Mathematics, 13 (1990), pp. $251-257$

36. M. KENNEDY, Characterizations of $C^{*}$-simplicity, 2015, arXiv: 1509.1870

37. A. LE BOUDEC, $C^{*}$-simplicity and the amenable radical, enventionesMathematicae, 209 (2017), pp. $159-174$

38. A. LE BOUDEC, AND N. MATTE BON, Subgroup dynamics and C*-simplicity of groups of homeomorphisms, 2016, arXiv: 1605.01651

39. T. OMLAND, Dynamical systems and operator algebras associated to Artin's representation of braid groups, 2016, arXiv: 1609.04737

40. N. OZAWA, Lecture on the Furstenberg boundary and C*-simplicity, http:// www.kurims.kyoto-u.ac.jp/ narutaka/notes/yokou2014.pdf, 2014

41. J. A. PACKER AND I. RAEBURN, Twisted crossed products of C*-algebras, Mathematical Proceedings of Cambridge Philosophical Society, 106 (1989), pp. 293 - 311

42. R. T. POWERS, Simplicity of the $C^{*}$-algebra associated with the free group on two generators, Duke Mathematical Journal, 42 (1975), pp. 151 - 15643

43. T. POZNANSKY, Characterizations of linear groups whose reduced $C^{*}$ algebras are simple, 2008, arXiv: 0812.2486

44. S. RAUM, C*-simplicity of of locally compact Powers groups, 2015, arXiv: 1505.0779. To appear in journal für die reine und angewandteMathematik

45. M. TAKESAKI, Theory of Operator Algebras I, Studies in Advanced Mathematics, SpringerVerlag New York Inc., 1979

46. R. D. TUSKER-DROB, Shift minimal groups, fixed price 1, and the unique trace property, 2012, arXiv: 1211.6395

47. W. A. WEECH, Topological dynamics, Bulleting of the American Mathematical Society, 83 (1977), pp. $775-830$

48. G. ZELLER-MEIER, Produitcroisésd'une C*-algébre par un grouped'automorphismes, Journal de MathématiquesPures et Appliquées. NeuviémeSérie, 47 (1968), pp. 101 - 239 